\title{
Study on the Interactive Strategy of Mixed Teaching Based on SPOC
}

\author{
Xiang Hua ${ }^{1, a^{*}}$, Chen Gang ${ }^{1, b}$ and Li Zhicheng ${ }^{1, \mathrm{c}}$ \\ ${ }^{1}$ School of Mathematics and Computer Science, Jianghan University, Wuhan, China \\ a*xianghua@jhun.edu.cn \\ ${ }^{b}$ Lao_chen@jhun.edu.cn \\ candersli@163.com
}

\begin{abstract}
Teaching interaction is an important factor which affects the quality of teaching. Reasonable and promptly interaction can improve the teaching effect. Teachers are the designers and leaders of teaching interaction. In the process of mixed teaching, teachers should combine various interactive methods to provide students with learning guidance in time. In order to enhance the quality of teaching interaction, improve the teaching effect, the paper analyzes online and offline interaction of mixed teaching based on SPOC, establishes the interaction model, indicates the interactive strategies, and analyzes the problems that should be paid attention to in the interaction process.
\end{abstract}

Keywords: Mixed Teaching, Instructor-Students Interaction, Interaction model, Interaction strategy

\section{基于SPOC混合教学的交互策略研究}

$$
\text { 向华 }{ }^{1, a^{*}} \text {, 陈刚 }{ }^{1, b} \text { ，李支成 }{ }^{1, c}
$$

${ }^{1}$ 江汉大学数学与计算机科学学院, 武汉, 中国

a*xianghua@jhun.edu.cn

${ }^{b}$ Lao_chen@jhun.edu.cn

candersli@163.com

\section{摘要}

教学交互是影响教学质量的重要因素, 合理及时的交互可以提高教学效果。教师是教学交互的设计 者、主导者, 在混合教学过程中要综合运用各种交互方式, 及时为学生提供学习指导。本文分析基于 SPOC 的混合教学中线上、线下交互过程, 建立交互模型, 设计交互策略, 分析交互过程中应注意的问 题, 以提高教学交互质量, 提升教学效果。

关键词: 混合教学，师生交互，交互模型，交互策略

\section{1. 引言}

交互可以理解为交互主体间相互影响、相互作用 并能引起对方的行为、性质、存在方式、价值观等发 生变化的任何过程。 ${ }^{[1]}$ 教学交互是一种发生在学生和学 习环境之间的事件，包括师生、生生之间的交流，也 包括学生和各种物化的资源之间的交流和相互作用 ${ }^{[2]}$ 。 教学交互贯穿于整个教学过程, 交互形式、交互频率、 交互质量是影响学习效果的重要因素。

近年来, 随着各大在线教学平台的兴起, 基于线 上教学平台的混合教学模式在越来越多的课程中采用。 在混合教学中, 教师在教学平台构建学习资源, 利用 各种线上教学工具对学生进行辅导, 监控学生学习情 况; 学生利用线上学习资源自主学习, 使用各种工具
进行讨论交流。因为很多传统教学中的知识点的讲授 任务转移到了线上, 混合教学中的线下课堂教学与传 统课堂教学相比发生了巨大变化。教师在线下课堂的 教学任务不再是知识点的讲授, 而是利用讨论、分组 项目协作、测试等各种方式了解学生实际学习情况, 培养学生对知识的综合运用能力、自主学习能力。

在混合教学过程中, 传统教学以师生课堂上同步 交互为主的交互方式发生了巨大变化，演变为线上、 线下的师生交互、生生交互、学生与学习内容交互、 教师与学习内容交互等多种交互类型并存，同步、异 步交互交织的多层次多维度交互。利用线上教学平台 和各种沟通工具, 师生、生生之间的交互距离大大拉 近, 交互频率明显提高。教学平台记录了大量的交互 数据, 教师能够根据交互数据对学生学习状态、学习 效果进行分析, 做出教学决策, 改进教学资源和教学 
方法。

\section{2. 混合教学中的交互模型}

在混合教学中, 教学过程根据教学内容、教学进 度划分为若干教学单元, 每个教学单元包含一个学习 章节, 或持续一段固定的教学时间。每个教学单元包 含线上课堂、线下翻转课堂两个阶段。每个教学阶段 都存在多种类型、多种形式的教学交互, 交互模型如 图 1 所示。因为每个教学阶段的侧重点不同, 采用的交 互方式和策略也不相同。

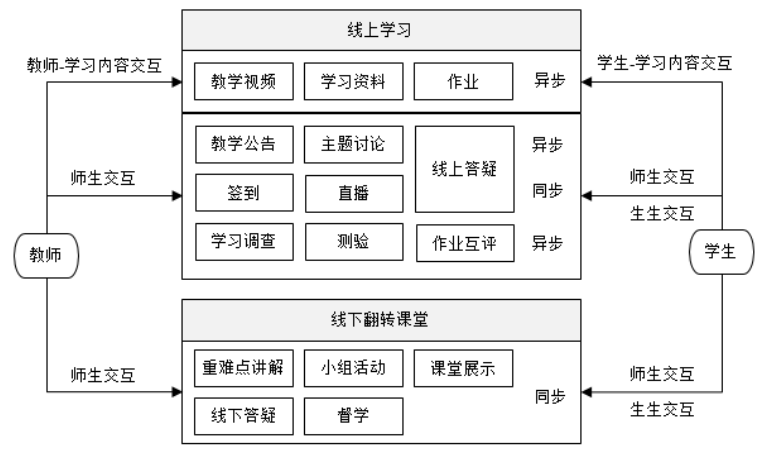

图 1 混合教学交互模型

\section{1. 线上学习阶段}

线上学习阶段由自主学习和线上课堂两部分组成。 在开始教学活动之前, 教师首先要分析学情, 根 据教学目标构建线上教学资源, 包括学习视频、PPT、 文档、作业等。这一部分交互是教师与学习内容之间 的交互, 交互过程简单。教师在设计学习内容时, 要 同时设计适当的学习活动, 即学习过程中的交互内容, 例如线上讨论话题、投票、测验等, 利用高质量交互 活动激发学生学习兴趣, 提高学生知识建构水平和学 习质量。

教学资源构建完成后, 教师发布教学公告, 布置 学习任务、学习要求。学生根据教师要求开始自主学 习, 与学习内容之间进行交互, 包括观看教学视频、 阅读学习资料、完成作业等。

为了保证学生有持续的学习兴趣和学习动力, 在 学生开始自主学习时, 线上的师生、生生异步交互也 同时开始。教师在线上发布与学习内容相关的主题讨 论, 学生在自主学习过程中自主参与。学生利用讨论 区、课程群讨论各种学习问题。教师对讨论内容进行 管理、评价, 并及时回复学生各种问题。

除了异步交互以外, 线上学习过程中也可以进行 同步交互。随着近两年各种直播平台的快速发展, 学 生对直播的接受度高, 参与直播互动的积极性也较高。 部分教学平台开发了直播功能, 教师通过直播可以实 时与学生交互, 为学生讲解教学重难点、答疑解惑, 及时了解学生的学习状态和学习情况。教师也可以根 据课程运行情况, 规定时间在线上进行实时主题讨论
和答疑，提高学生参与度，使讨论和答疑更具时效性。 在教学单元结束阶段, 教师通过教学平台发布测 验、学习调查, 分析学生学习数据、讨论情况、测验 成绩和学习调查结果了解学生学习进度、学习效果, 根据学生情况设计线下课堂内容和活动, 根据学生反 馈调整教学方式、教学资源, 对未完成学习任务的学 生督学, 使学生达到课程基本学习要求。

在整个混合教学流程中, 教师利用教师一学习内容 交互构建教学内容, 设计交互内容和交互方式, 通过 观察线上学生一学习内容交互、师生交互和生生交互, 对线上学习资源进行进一步完善和修改, 并设计线下 课堂交互活动，形成多种形式交互交叉迭代。

\section{2. 线下翻转课堂阶段}

学生完成线上课堂学习任务后, 对教学内容已基 本掌握, 进入线下翻转课堂阶段。在线下翻转课堂中, 教师对线上学习情况进行分析和总结, 梳理重难点, 通过组织小组活动引导学生灵活运用所学知识解决实 际问题。

在线下翻转课堂阶段, 所有师生、生生交互为同 步交互。因为课堂上不再讲授知识点, 所以以教师为 主导的单向交互大大减少，教师大部分教学工作转变 为参与、观察学生活动, 了解、评价学生学习情况, 与学生讨论交流。虽然线下翻转课堂在整个教学过程 中一般只占用三分之一至二分之一学时, 但这一阶段 培养学生综合运用能力、创造力、团队合作能力, 是 引导学生达到课程高阶要求的重要阶段。

目前一些教学平台也提供线下课堂的实时交互工 具，例如课堂选人、抢答、投票、实时讨论、投屏等。 这些交互工具可以方便教师在课堂上使用手机与学生 开展各种活动, 同时也记录交互数据, 为教师提供更 精确的教学参考。

\section{3. 交互策略}

在混合教学中, 师生交互、生生交互、学习内容 交互等各类交互的参与性和交互频率都比传统教学有 较大提升。采取积极的交互策略, 营造良好的交互氛 围有利于促进各类交互, 提高学生学习兴趣和参与度, 保证学习质量和学习满意度。

\section{1. 提高学习内容交互频度}

在混合教学中, 学生-学习内容交互自主进行。学 习资源的难度、趣味性、时长直接影响学生的学习效 果。教师在设计教学内容时应该根据学情设置稍高于 学生学习能力的教学内容, 既能保证一定的高阶性, 也能让学生在学习过程中有成就感、获得感, 激发学 习兴趣。因为高频率、短时长的交互更能吸引学生兴 趣, 所以教学视频时长控制在 10 分钟左右或更短较好。 
教师根据知识点尽量将教学视频拆分成小段, 并在视 频播放过程中加入与视频内容相关的少量测验题, 使 学生始终与学习内容保持较高的交互频度, 减少刷视 频现象。

教师与学习内容的交互是混合教学交互过程中最 早进行的交互, 教师建立教学资源后, 在学生学习过 程中还应根据线上、线下课堂师生交互中发现的问题, 针对学生能力、需求和学习状态修改完善学习资源, 补充学习资料, 取得更好的教学效果。

\section{2. 保持异步交互时效性}

在传统教学活动中, 教师处于权威地位, 学生必 须服从教师的管理。而线上学习虽然拉大了师生之间 的物理距离, 但因为线上学习不受时间、空间限制, 学习氛围更加宽松, 师生之间心理距离反而拉近, 学 生交互意愿、交互频率明显高于传统课堂。在线上交 互过程中, 大部分师生、生生交互是异步交互, 存在 一定时间的延迟。对于学生提出的问题, 参与的讨论, 教师快速的响应不仅能及时帮助学生解决各种学习问 题, 促进学生更深层次的思考, 对班级其他学生也能 起到鼓励示范作用，促使学生更积极的参与各种交互。 由于学生在生活中更习惯于利用各种社交工具例如 $\mathrm{QQ}$ 、 微信交流信息, 在进行交互时可以将教学平台的讨论 区与社交媒体结合使用，进一步提高交互时效性。

\section{3. 提高生生交互积极性}

而在线上学习过程中, 生生交互比传统课堂更加 自由, 学生更愿意表达自己的观点。在学生适应线上 学习的基本流程后, 学生在教学平台和社交工具中的 相互交流频次明显增加, 不善表达和内向的学生能通 过网络提问和发表观点, 学习较好的学生乐意帮助同 学解决各种问题。教师可以对积极参与讨论和帮助同 学的学生给予奖励, 或者设置学生助教, 鼓励生生互 动, 逐步引导学生成为课程讨论区、学习群中的交流 的主体，创建深层次互助探讨的学习氛围。

线上互评活动是同伴学习中的一种交互策略, 学 生通过互评工作对同学进行指导和评价。通过在课程 中组织多次互评活动中发现, 学生对担任导师角色, 拥有评阅权利感兴趣, 大部分学生认真检查同伴作业, 公正指出存在的问题并评分。在同伴指导过程中, 作 为导师角色的学生需要对所学内容有更深刻的理解, 才能针对同伴提出问题、解释问题。学生在互评过程 中遇到问题也会更积极的讨论、思考, 或主动从教师 处寻求帮助, 从而发现自己在学习过程中忽略的问题, 进一步加深对学习内容的理解和掌握。为了保证互评 活动公平，教师应提供详细评阅标准，设置互评时评 阅者和被评阅者互相隐藏身份, 及时回应评阅争议, 使互评活动能健康持续进行。

\section{4. 设计线下课堂交互内容}

在混合教学中, 线下课堂的主要任务包括两方面: 一是帮助学生解决线上学习中的问题; 二是引导学生 对所学知识进行综合运用。

在教学资源设计阶段, 教师就要根据教学内容预 先设计线下课堂的小组活动任务。小组任务应是综合 性、创新性内容, 难度和工作量应高于线上学习任务, 学生经过查阅资料、讨论、协作能完成。在布置学习 任务时，小组活动任务与线上学习任务一同布置，使 学生有足够的时间讨论、完成。

在线上学习阶段, 教师要观察分析线上学生与学 习内容交互、师生交互、生生交互中出现的问题, 归 纳整理在线下课堂讲解的内容。在线下课堂活动中, 教师要及时帮助学生解决小组任务时遇到的问题, 选 择优秀项目、有共性问题的项目进行展示、讨论和点 评。

因此, 在每一个教学单元, 教师需要在布置学习 任务之前和线下课堂上课前进行线下课堂的内容和交 互设计。积极、热烈的线下课堂交互能进一步提升学 生的学习兴趣和学习效果, 促进更多学生达到课程高 阶教学要求。

\section{5. 加强交互监督管理}

不论是在线上还是线下, 教师应对学生交互进行 及时的监督管理。由于学生性格不同, 学习能力不同, 在交互过程中会存在少数沉默者和边缘人。教师可以 根据教学平台的交互数据及时发现这些学生, 鼓励学 生选择合适的表达方式参与互动, 逐步转变学生不良 学习习惯。

另外, 也有少数学生为了提高教学平台记录的学 习数据在讨论区发布水贴, 为了应付学习任务刷学习 视频, 抄袭作业。教师应该采取严格的管理措施, 例 如对水贴扣分, 在学习视频中间加入测试题, 严惩雷 同作业，促使班级中形成正向、有益的交互氛围，培 养学生良好的学习习惯, 引导学生逐步进入深层次学 习。

\section{4. 实施效果}

本交互模型 2018 年在本校《大学计算机基础》课 程混合教学中广泛使用, 线上自主学习、线上课堂与 线下课堂学习时间比例为 $1: 1: 1$ 。学生在教学平台自主 学习, 教师发布学习任务, 引导学生在线上进行讨论 交流, 利用定期直播与学生实时沟通, 观察学生交互 过程并进行监督。在线下课堂, 教师对学生学习中的 重难点进行讲解, 开展课堂讨论和测试, 检查学生学 习效果。2019 年, 课程混合教学中进一步完善交互策 略, 在每一章增加线上主题讨论内容, 要求学生全体 参与, 引导学生自主查阅资料和思考, 在综合性作业 
中开展生生互评活动, 促使学生对所学内容进行回顾 和思考。在线下课堂中开展 PBL 项目小组活动, 学生分 组完成项目并在线下课堂展示, 教师点评并进行小组 互评, 线下课堂学生反应热烈。采用混合教学交互模 型并完善交互策略后，两年的期末考试成绩明显提升。

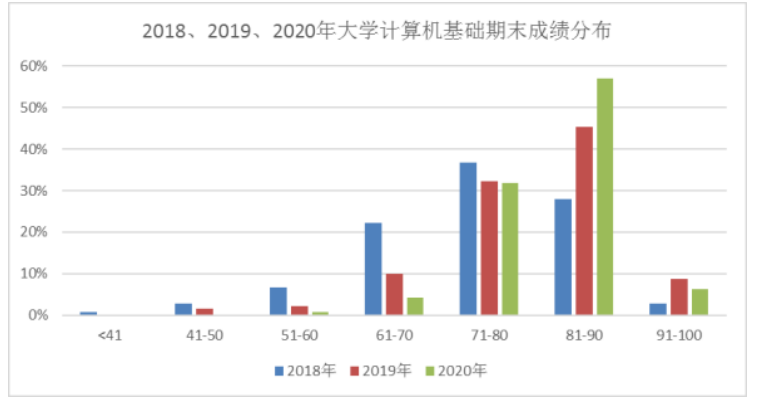

图 2 2017、2018、2019 年大学计算机基础期末成绩 对比

2020 年春，因新冠疫情原因《Flash动画制作》课 程临时由传统课堂教学转为全线上教学, 教学过程中 运用本交互模型中的线上部分和交互策略, 也取得了 良好的教学效果。

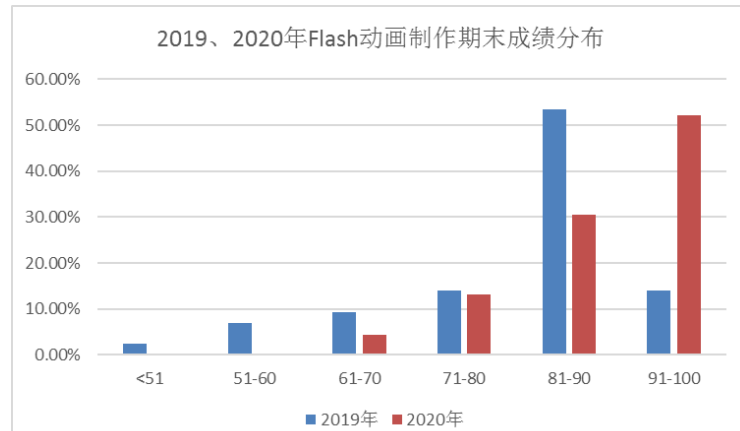

图 3 2019、2020 年 Flash 动画制作期末成绩对比

\section{5. 小结}

经过实践, 可以看出本交互模型和交互策略能提 高学生学习兴趣, 促进混合教学过程中的各类交互活 动, 取得更好的教学效果, 可以推广到其他混合教学 课程中。

本交互模型在实施时, 需要教师全过程积极参与, 并且要有丰富的教学经验和较强的教学组织能力。教 师进行交互设计, 观察并引导学生进行各种交互的工 作量非常大, 需要投入大量精力和时间。教学平台虽 然记录了大量交互数据, 但对数据的分析工作主要依 靠教师手工进行。如果使用人工智能对交互数据进行 分析处理, 为教师推送分析结果, 将减轻教师工作量, 使教师专注于交互内容和交互活动的设计, 将取得更 好的教学效果。

\section{项目基金}

本文为武汉市教育科学 “十三五” 规划 2018 重点课题《基于 SPOC 的混合教学交互过 程研究》（2018A047）、江汉大学校级教学 研究重点项目《基于 SPOC 混合教学的学习评 价体系研究》（2018X003）的阶段性成果之 一。

\section{REFERENCES}

[1] HAN Qin, ZHOU Zong-Kui, HU Wei-ping, The Influential Factors of Classroom Interaction and Teaching Revelation. Theory and Practice of Education, 2008, Theory and Practice of Education, 2008, (6) :42-45.

[2] Chen Li, An Investigation into "Interactivity" and the Related Concepts[J]. Distance Education in China, 2004(3):12-16.

[3] Lv Linhai, Gong Fang. A Comparative Research on Undergraduates' Deep Learning and Its Influence Mechanism between the Research Universities in China and USA_— Based on the SERU Data Analysis for the Eight Universities in China and USA, Educational Research, 2018,(4):111-120.

[4] Hua XIANG, Gang CHEN and Zhi-cheng LI, Research and Practice on Hierarchical Supervisory Strategy of Mixed Teaching. 2019 International Conference on Management Innovation, Education Reform and Applied Social Science (MIERASS2019), 2019.4

[5] CHEN Beilei, ZHANG Yi, YANG Bing, XIONG Jie, LIN Li. Study on Effects Of Instructional Interaction on Deep Learning in Smart Classroom[J]. e-Education Research, 2019,(3):90-97 